\title{
The use of OMA for the validation of the design of the Allianz Tower in Milan
}

\author{
Elena Mola ${ }^{(1)}$ Franco Mola $^{(2)}$ Georgios Stefopoulos $^{(3)}$ Carlo Segato $^{(4)}$ Chiara Pozzuoli $^{(5)}$ \\ ${ }^{(1)} \mathrm{CEO}$ and ${ }^{(3)}$ Senior Engineer \\ ECSD Srl, Via Carlo Goldoni, 2220129 Milan \\ (4) P. Structural Engineer, Milan \\ (2) Full Professor \\ Politecnico di Milano, Piazza Leonardo da Vinci, 3220133 Milan \\ (5) Project Manager, \\ RWDI, Lawrence Industrial Estate, Lawrence Way, Dunstable \\ LU6 1BD, United Kingdom
}

\begin{abstract}
Future headquarter of an insurance company, the Allianz Tower is a $202 \mathrm{~m}$ high, 52 -storey building currently topped out and belonging to the large CityLife development in Milan, Italy. The architectural design concept, by Arata Isozaki, is that of a slender, streamlined machine-building, with exposed structural and functional systems. The very significant slenderness and the intrinsic damping properties of the Tower make lateral and torsional response due to wind actions not negligible for structural analysis and occupant comfort. To mitigate these effects eight external viscous dampers were designed,and located at the base of four steel "struts", stemming out of the surrounding plaza and podium at the base of the tower and connected to its main cores at the 11th floor. The design of the dampers and of the struts required refined analyses to be carried out. At the end of construction, two sets of Operational Modal Analysis (OMA) tests were carried out on the building, with and without dampers, to validate the design and construction process. Moreover, given the importance of the building, the Tower was equipped with a state-of-the-art continuous monitoring system acquiring data on all of the relevant structural and functional parts, including the dampers. The present paper will present the main structural features of the Tower, the main features of the design of the damping devices for wind comfort, the test set up for the dynamic OMA tests, followed by a discussion of their results.
\end{abstract}

KEYWORDS: Operational Modal Analysis, Tall Building, External Dampers, Model Tuning

\section{Introduction}

In Italy, dynamic tests on buildings are not explicitly required by the current Building Code, i.e. NTC 2008, [1]: the Code only states that for 'strategically relevant' bridges and flyovers, some kind of dynamic testing is required in addition to traditional static load tests. Nevertheless, for important buildings, and tall buildings in particular, it is widely accepted by practitioners and contractors alike that dynamic tests are a very valuable source of information that cannot be derived from any other kind of test. In fact, by means of experimental or operational modal analysis, experimental evidence of the global behavior of the structure is obtained, in particular as for seismic and wind excitation response, the dynamic properties of the building are measured, in terms of frequencies and mode shapes, and compared to those derived from numerical analysis. Through this process, numerical models are validated and a benchmark for continuous monitoring activity is obtained, so that maintenance operations can be planned and decisions about the operability of the building can be quickly made in the aftermaths of exceptional events. Because dynamic tests provide such valuable information, in the case of tall buildings, which are important and expensive structures, in Italy they are normally prescribed by the Owner or by the project Validator 
so that they become a necessary pre-requisite in order for the design and construction process to be validated, even if they are not mandatory according to the Italian Building Code.

This was the case for the Allianz Tower: both the project Validator and the Owner agreed on the opportunity of carrying out dynamic tests at the end of construction: it was decided to use the Operational Modal Analysis method, so that only the environmental excitation would be exploited to derive the modal properties, with no need to move and install large tools at the top of the building to provide additional forcing, which would have been required for Experimental Modal Analysis, [2].

The results of the dynamic tests were used to validate the finite element model of the building that had been implemented in the structural analysis software MidasGen. Moreover, since a peculiar architectural and functional feature of the Allianz Tower remains in the external viscous dampers at the base of the struts, which are designed to provide additional damping under wind loads, i.e. in service life conditions, for very low levels of vibration, the OMA tests in this case also provided insight on the actual efficiency of those devices: the tests were carried out before and after the installation of the dampers, so that it was possible to experimentally derive the modal damping in service life conditions with and without the contribution of the dampers, thus obtaining important data for the validation of their design and installation process.

\section{Description of the structure}

The Allianz Tower occupies the center of the redevelopment site of the former exhibition center in Milan, called CityLife, in front of the building that housed the old Sports Palace, which overlooks Viale Boezio. The building, which is going to be used as the headquarters offices of Allianz in Italy, has a height of $202 \mathrm{~m}$ above the ground level of the main plaza and $207 \mathrm{~m}$ above the street level. Below the ground level, one underground level connects the tower with the underground shopping area as well as the metro station, and two other underground levels are devoted to mechanical use.

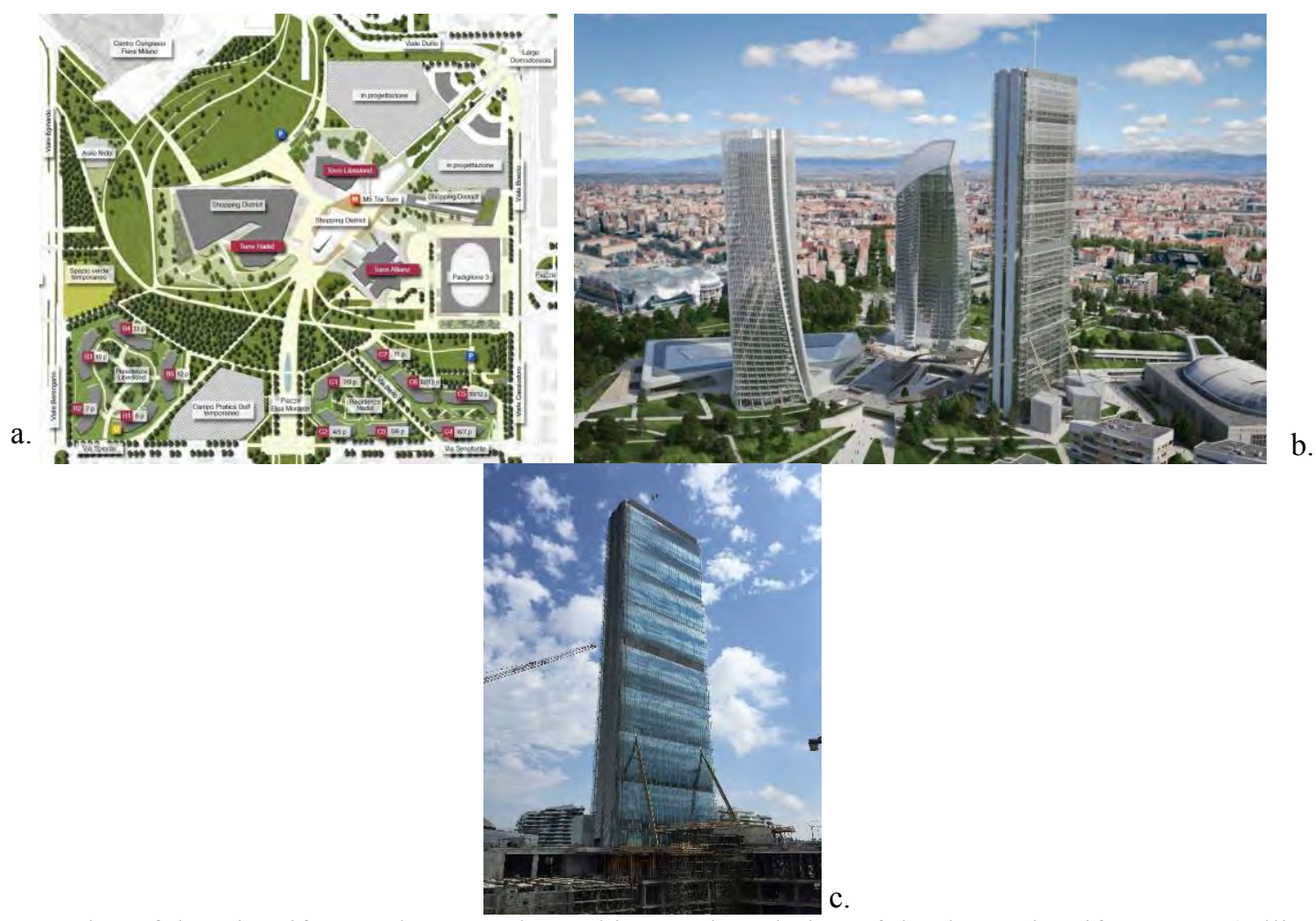

Fig.1 - a. Masterplan of the CityLife Development; b. Architectural rendering of the three CityLife Towers (Allianz Tower is on the right), c. The topped-out Allianz Tower

The structural system is composed of two main reinforced concrete cores, with a wall thickness varying from $120 \mathrm{~cm}$ to 40 $\mathrm{cm}$, located on the North and South sides of the tower; two outrigger structures, called belt-trusses, connect the two cores. The first belt truss is a two-storey high steel truss structure placed mid-height, i.e. between Levels 23 and 26, whereas the 
second one is a prestressed reinforced concrete wall beam, placed at the top of the building, i.e. between Levels 49 and 50 . Finally, four external steel struts, covered in golden paint, jutting out of the building at mid-height, connect it to the ground, at the top of the podium: at the base of each strut, two two-way viscous dampers are installed, which help to mitigate the effects of the resonant component of the wind excitation, thus improving the comfort of the building.

The columns have circular sections, with diameters ranging from 170 to $65 \mathrm{~cm}$. Up to Level 4 for the external columns, and up to Level 21 for the internal ones, the vertical elements are composite steel-concrete columns, having a circular reinforced concrete section with a composed cross-shaped steel section inside. The decks are $20 \mathrm{~cm}$ thick, cast-in-situ reinforced concrete slabs supported by T-beams, having a depth of $45-50 \mathrm{~cm}$. In Fig.2, some details of the structural design of the building are provided: a typical floor plan, the scheme of the concrete classes used for the structural elements and a view of the composite cross section of the columns at the lower levels.

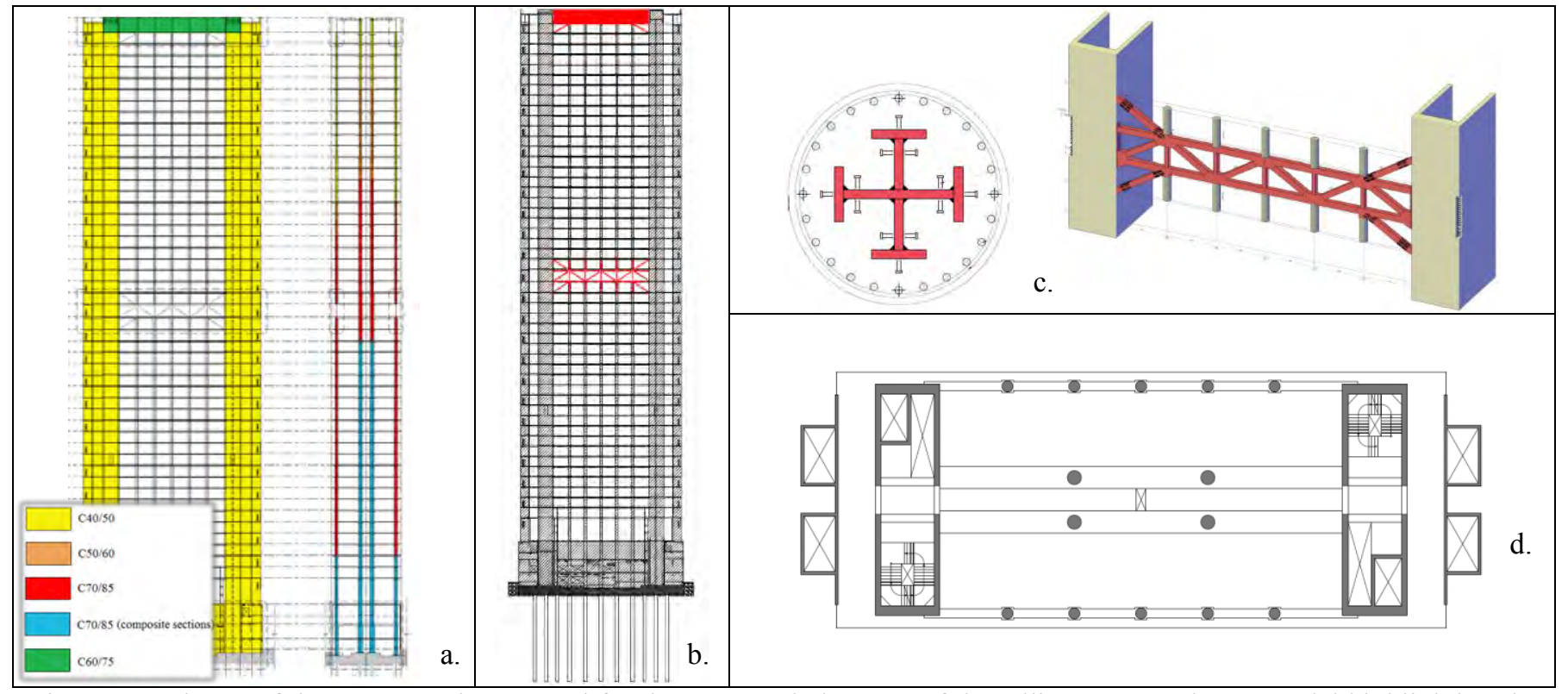

Fig. 2 - a. Scheme of the concrete classes used for the structural elements of the Allianz Tower, b. FE model highlighting the belt trusses c. Typical cross-section of the composite columns at the lower levels and view of the steel belt-truss, d. Typical plan of the Allianz Tower

\section{Modeling and analysis}

\subsection{Modeling Assumptions}

The dynamic properties of Allianz Tower were numerically analyzed by means of a finite element (FE) model which was developed using the commercial software for structural analysis MidasGen 2012 V3. Since the main purpose of this model is to provide a numerical counterpart for the experimental results of the operational modal analysis, great care was taken in accurately replicating the actual longitudinal and transverse geometry of the structure and its loads and boundary conditions at the time of the dynamic tests.

The FEM model itself is made of 12.000 linear elements, which are mostly used to represent the columns, the belt trusses and some secondary elements, and 105.000 plate elements, which were used to model not only the decks and the concrete cores, but the T-beams of the decks as well.

This modeling choice for the beams proved to yield more accurate results with respect to more simplified models that had been implemented as a benchmark: due to the fact that the depth of the beams is only $0.5 \mathrm{~m}$, i.e. not far from the depth of the slabs, which is $0.2 \mathrm{~m}$, and because the spans are significant, in the range of 6 to $8 \mathrm{~m}$, the global behavior of a single floor is very close to that of a bending membrane plate, which is better approximated with the use of plate elements for both the 
beams and the slab. Due to the slenderness of the structure and of the slabs, no floor diaphragm constraint was applied for the floors, which can freely bend and deform out of plane.

As for the eigenvalue analysis itself, the calculation of eigenvectors was carried out by the method of Lanczos and, for all the elements of the structure, the geometry of the gross cross section was considered, since the structure is expected to behave elastically under the low dynamic loads provided by the environmental excitation. The composite sections of the columns of the first floors was represented in the model with homogenized sections with respect to their bending moment stiffness (EI). Finally, an eigenvalue analysis was carried out for both the building with and without the struts and the dampers. . Due to the negligible effect of the struts on global stiffness and mass of the building, the results of the eigenvalue analysis, in terms of frequencies and mode shapes, were identical for both cases, as expected.

The same model was used to carry out a dynamic response spectrum analysis. The spectra used for the analysis are specifically derived for slender building, i.e. for periods larger than $4 \mathrm{~s}$, and the damping values used were those compatible with a very streamlined tall building, i.e. $1 \%$ for all the modes, instead of $5 \%$. The expected low values of inherent damping were then increased by introducing the additional damping contribution that the dampers were designed to provide, i.e. a nominal additional damping of $9 \%$ on the first and on the third mode. As further detailed in the following, one of the results of the dynamic tests was to confirm the estimation both of the inherent and of the additional damping, thus validating the design and installation of the dampers themselves.
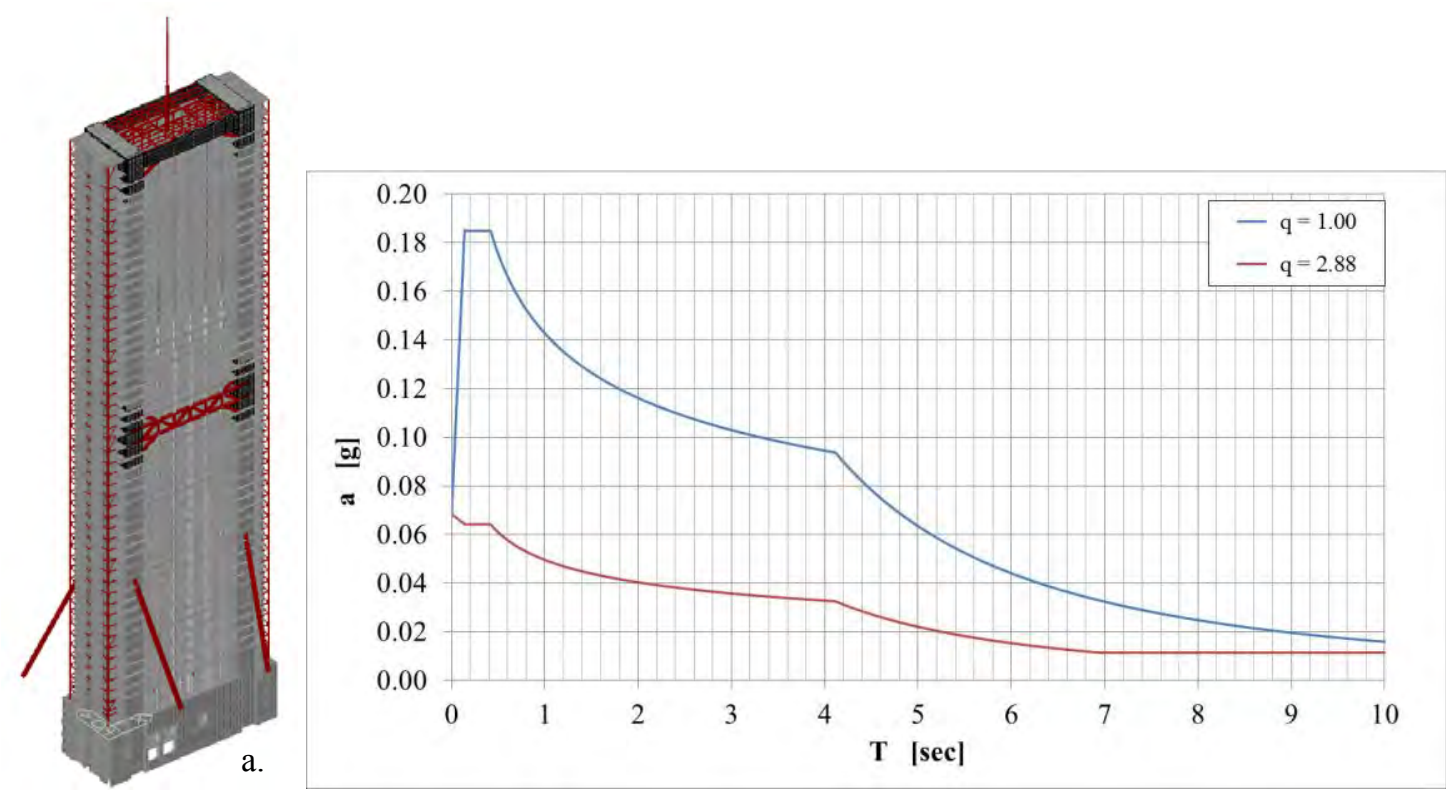

b.

Fig.3 - a. Finite element model of the Allianz Tower for eigenvalue and response spectrum analysis; b. Site-specific, longperiod response spectrum used for the analysis

\subsection{Eigenvalue Analysis results}

In the following, the frequencies and mode shapes obtained from the numerical model are reported. In Fig.4a-c), the first twelve mode shapes are represented, whereas in Tab.1, the modal masses, frequencies and periods are summarized. Only the results pertaining to the configuration without struts and dampers are reported, since, as discussed before, the results of the analysis on the final configurations are not affected by the presence of the struts and dampers, which provide only negligible contributions in terms of additional mass and stiffness. From Fig.4 and Tab.1, it can be noted that the first mode is mostly flexural along the weak axis of the building, the second mode is mostly flexural along the strong axis, whereas the third mode is mostly torsional. Since the building is very slender in the weak direction, it is particularly sensitive to the across-wind effects when the wind blows along the long side of the building; also, the sensitivity to wind loads along the weak axis is enhanced by torsional effects, which are evident already in the third mode. 

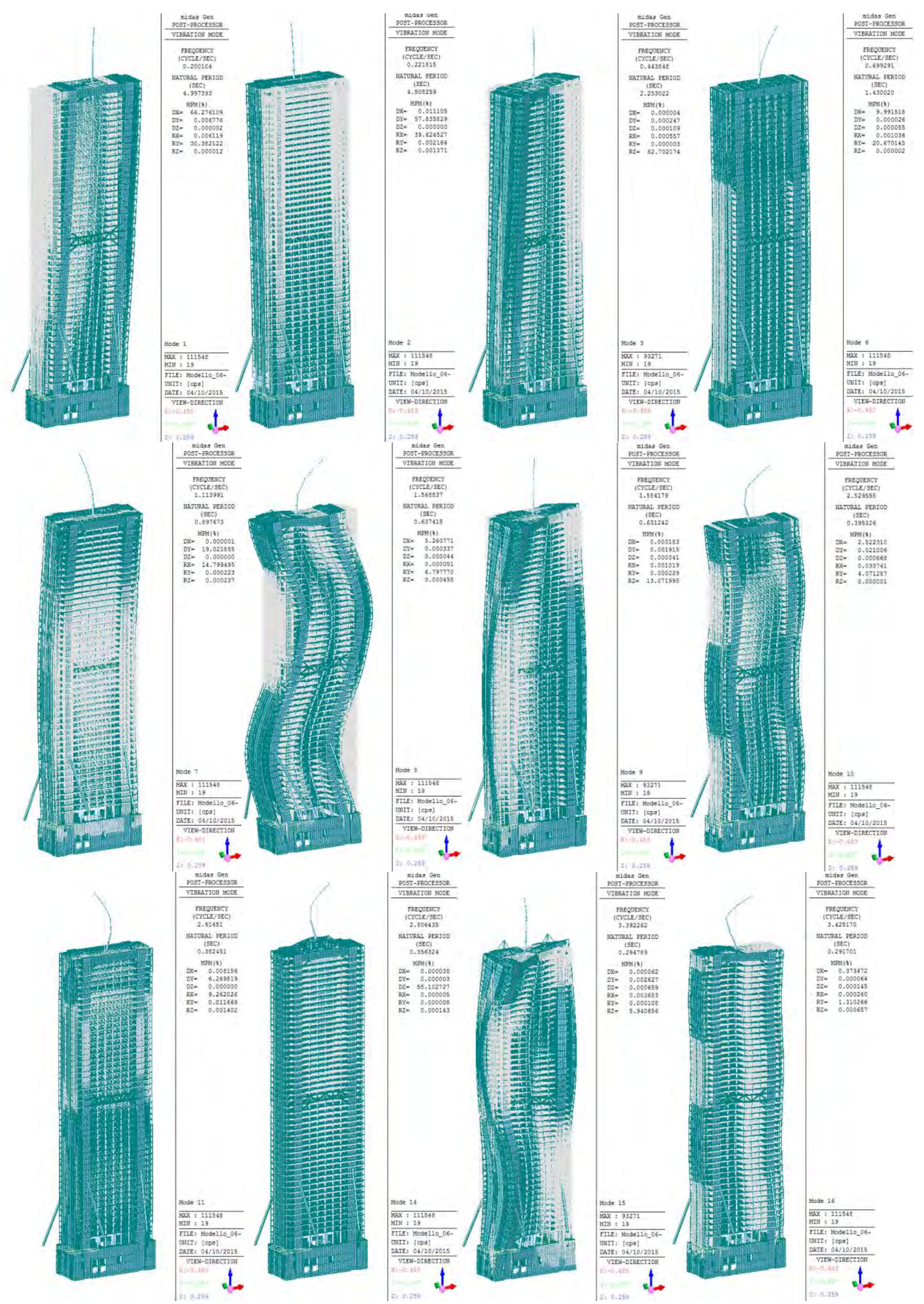

Fig. 4 - Mode shapes of the first 12 vibration modes of the Allian Tower, as derived by eigenvalue analysis 
Tab.1 - Frequencies, periods and modal mass values for the first 20 vibration modes of the Allianz Tower

\begin{tabular}{|c|c|c|c|c|c|c|c|c|c|}
\hline \multirow{2}{*}{ Mode No } & \multicolumn{2}{|c|}{ Frequency } & Period & \multicolumn{2}{|c|}{ TRAN-X } & \multicolumn{2}{c|}{ TRAN-Y } & \multicolumn{2}{c|}{ TRAN-Z } \\
\cline { 2 - 10 } & (rad/sec) & (cycle/sec) & (sec) & MASS(\% & SUM(\%) & MASS $\%$ & SUM(\%) & MASS(\% & SUM(\%) \\
\hline 1 & 1.26 & 0.20 & 5.00 & 66.28 & 66.28 & 0.01 & 0.01 & 0.00 & 0.00 \\
\hline 2 & 1.39 & 0.22 & 4.51 & 0.01 & 66.29 & 57.85 & 57.86 & 0.00 & 0.00 \\
\hline 3 & 2.79 & 0.44 & 2.25 & 0.00 & 66.29 & 0.00 & 57.86 & 0.00 & 0.00 \\
\hline 4 & 4.39 & 0.70 & 1.43 & 10.21 & 76.49 & 0.00 & 57.86 & 0.00 & 0.00 \\
\hline 5 & 7.00 & 1.11 & 0.90 & 0.00 & 76.49 & 19.03 & 76.89 & 0.00 & 0.00 \\
\hline 6 & 9.86 & 1.57 & 0.64 & 5.26 & 81.76 & 0.00 & 76.89 & 0.00 & 0.00 \\
\hline 7 & 9.96 & 1.58 & 0.63 & 0.00 & 81.76 & 0.00 & 76.89 & 0.00 & 0.00 \\
\hline 8 & 15.90 & 2.53 & 0.40 & 2.53 & 84.29 & 0.02 & 76.91 & 0.00 & 0.00 \\
\hline 9 & 16.47 & 2.61 & 0.38 & 0.01 & 84.30 & 6.59 & 83.50 & 0.00 & 0.00 \\
\hline 10 & 17.67 & 2.81 & 0.36 & 0.00 & 84.30 & 0.00 & 83.50 & 58.53 & 58.54 \\
\hline 11 & 21.32 & 3.39 & 0.29 & 0.00 & 84.30 & 0.00 & 83.51 & 0.00 & 58.54 \\
\hline 12 & 21.52 & 3.43 & 0.29 & 0.37 & 84.67 & 0.00 & 83.51 & 0.00 & 58.54 \\
\hline 13 & 23.77 & 3.78 & 0.26 & 1.93 & 86.60 & 0.00 & 83.51 & 0.01 & 58.54 \\
\hline 14 & 24.38 & 3.88 & 0.26 & 0.00 & 86.60 & 0.00 & 83.51 & 15.49 & 74.03 \\
\hline 15 & 26.03 & 4.14 & 0.24 & 0.00 & 86.60 & 0.00 & 83.51 & 0.02 & 74.05 \\
\hline 16 & 27.01 & 4.30 & 0.23 & 0.00 & 86.60 & 3.18 & 86.69 & 0.00 & 74.05 \\
\hline 17 & 27.49 & 4.38 & 0.23 & 0.00 & 86.60 & 0.01 & 86.70 & 0.01 & 74.06 \\
\hline 18 & 29.99 & 4.77 & 0.21 & 0.66 & 87.26 & 0.00 & 86.70 & 0.00 & 74.06 \\
\hline 19 & 31.19 & 4.96 & 0.20 & 0.00 & 87.26 & 0.00 & 86.70 & 0.00 & 74.06 \\
\hline 20 & 31.25 & 4.97 & 0.20 & 0.00 & 87.26 & 0.00 & 86.70 & 0.00 & 74.06 \\
\hline
\end{tabular}

\section{Experimental Analysis}

\subsection{Description of the tests}

The experimental tests were carried out according to the Operational Modal Analysis method, both before and after the installation of the viscous dampers. In both cases, the accelerations due to environmental excitation were continuously acquired for 3.5 days, with a sampling frequency of $20 \mathrm{~Hz}$, and averaged over three-hour windows.

The test set-up consisted of 30 horizontal accelerometers, measuring X- and Y-direction acceleration, according to the reference system in Fig.5, and 3 vertical accelerometers, measuring Z-direction accelerations. The location of the sensors is reported in Fig.5, represented the plan configuration of the typical instrumented floor. The same configuration was replicated for all the instrumented floors, i.s. Levels 12, 24, 33, 42, 49.

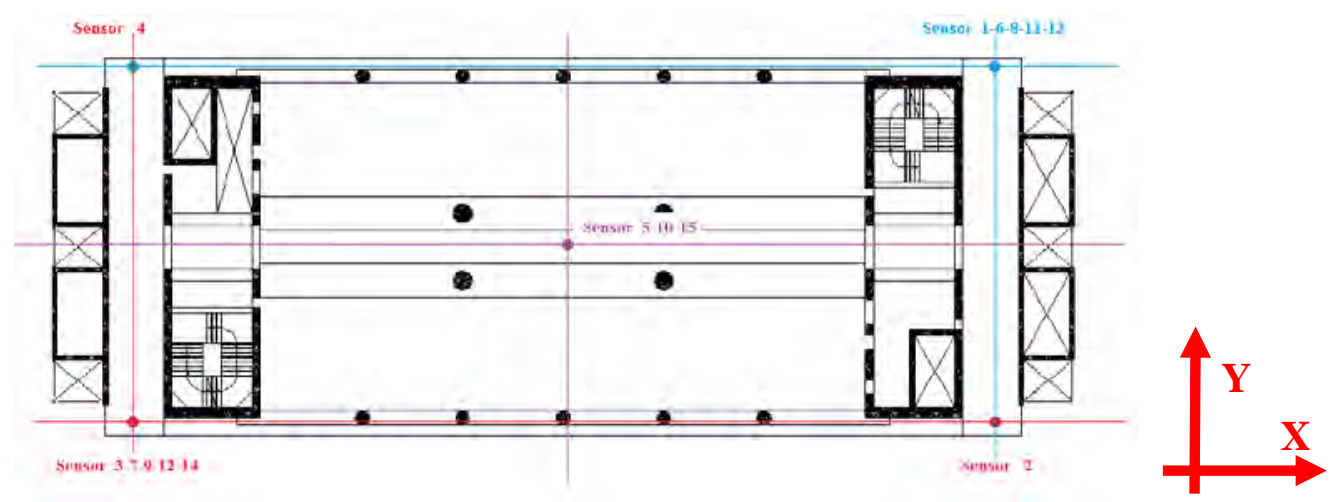

Fig.5 - Test set-up: location of the sensors on the typical instrumented floor

The accelerometers used for the tests were PCP Piezotronics sensors having high sensitivity $(10 \mathrm{~V} / \mathrm{g})$ and antialiasing filters. The wind speed was also measured by means of an anemometer directed along the $\mathrm{X}$ direction, according to the reference of Fig.5.

The data were analyzed by means of the Polymax algorithm, [3], implemented into an in-house software owned by the Company who carried out the tests, which automatically chooses the best averaging windows to minimize bias to the data due to the presence of workers or other accidental forcing sources. 
The PSD (Power Spectral Density) plots for some of the sensors are reported in Fig.6a.
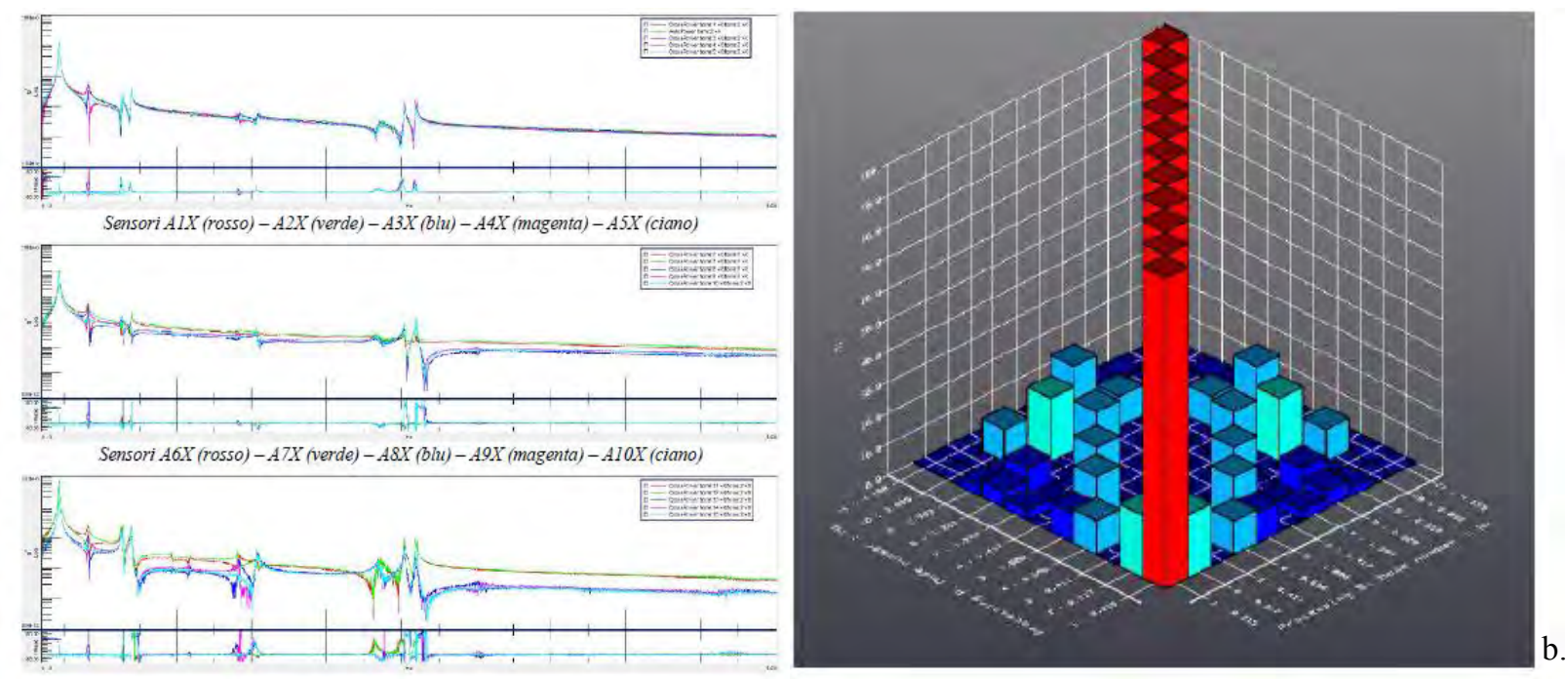

Fig.6 - a. PSDs for sample channels; b. AutoMAC matrix

In order to increase the reliability of the estimated modal properties, cross-correlation was checked between all the computed modes up to $5 \mathrm{~Hz}$, by calculating the values of the AutoMAC index, as exemplified in Fig.6b, showing the results for Mode 1.

\subsection{Test results and comparison with numerical analysis}

The experimentally derived frequencies and modal damping values are reported in Tab.2, together with the frequencies derived from the numerical model, for the modes that were identified: the numbering of the mode sequence is that of the experimental measures, but in brackets the numbering corresponding to the sequence of the eigenvalue analysis is also provided. In Fig.7-9, the experimental mode shapes of the identified modes are plotted together with the mode shapes derived from the numerical analyses for the first 9 identified modes. In order to meaningfully compare the mode shapes and to plot the values, the values of the eigenvectors from the numerical model were computed at the exact locations were the accelerometers were installed.

Tab.2 - Experimental and numerical frequencies and experimental damping estimates for the identified modes

\begin{tabular}{|c|c|c|c|c|}
\hline $\begin{array}{c}\text { Experimental } \\
\text { MODE \# (Numerical } \\
\text { MODE \#.) }\end{array}$ & $\begin{array}{c}\text { FREQUENCY [Hz] } \\
\text { (Exp.) }\end{array}$ & $\begin{array}{c}\text { FREQUENCY [Hz] } \\
\text { (Num.) }\end{array}$ & $\begin{array}{l}\text { Num. Freq./ } \\
\text { Exp. Freq. }\end{array}$ & $\begin{array}{l}\text { DAMPING [\%] } \\
\text { (Exp.) }\end{array}$ \\
\hline Mode N.1 (N.1) & 0.215 & 0.20 & 0.93 & 0.50 \\
\hline Mode N.2 (N.2) & 0.217 & 0.22 & 1.01 & 0.48 \\
\hline Mode N.3 (N.3) & 0.411 & 0.44 & 1.07 & 0.35 \\
\hline Mode N.4 (N.4) & 0.696 & 0.70 & 1.005 & 0.30 \\
\hline Mode N.5 (N.5) & 1.006 & 1.11 & 1.10 & 0.52 \\
\hline Mode N.6 (N.7) & 1.417 & 1.58 & 1.11 & $\mathbf{0 . 3 0}$ \\
\hline Mode N.7 (N6) & 1.544 & 1.57 & 1.02 & 0.34 \\
\hline Mode N.8 (N.9) & 2.329 & 2.61 & 1.12 & 0.60 \\
\hline Mode N.9 (N.8) & 2.353 & 2.53 & 1.07 & 0.24 \\
\hline Mode N.10 (N.11) & $\mathbf{3 . 0 3 5}$ & 3.39 & 1.1 & 0.29 \\
\hline
\end{tabular}

In Fig.10, the values of frequencies identified during the tests without dampers are plotted again and compared to those derived from numerical analysis and those identified during the tests with dampers: it can be noted that the presence of the dampers does not significantly affect the frequencies, as expected and as predicted by the numerical analyses. 


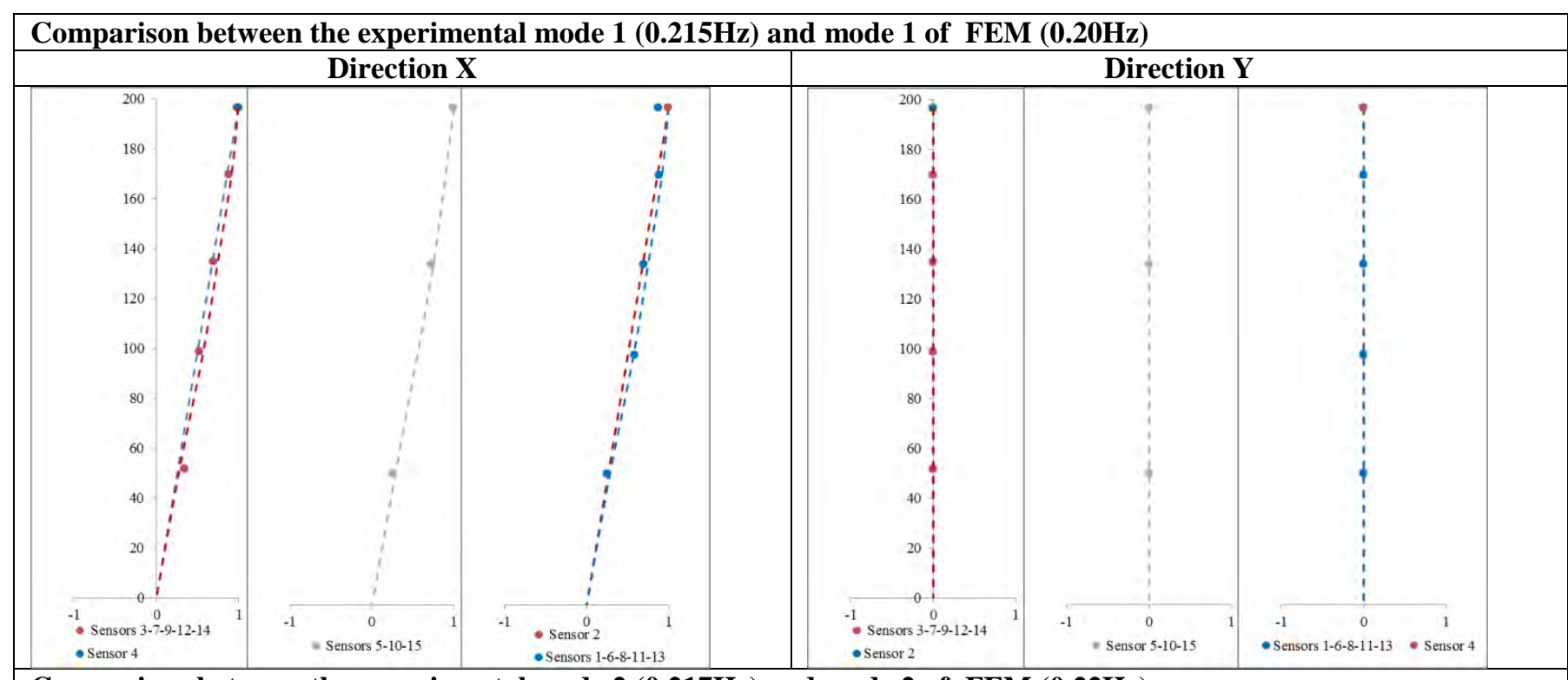

Comparison between the experimental mode $2(0.217 \mathrm{~Hz})$ and mode 2 of FEM $(0.22 \mathrm{~Hz})$

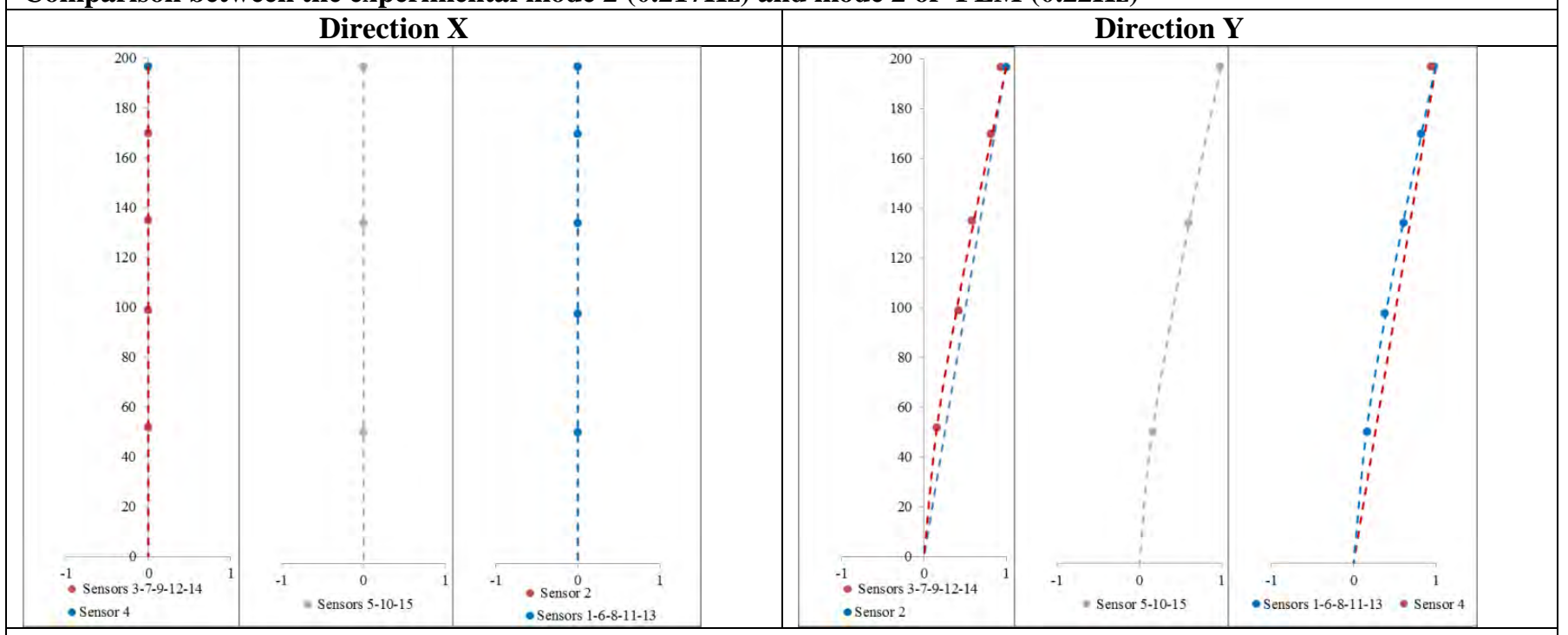

Comparison between the experimental mode $3(0.411 \mathrm{~Hz})$ and mode 3 of FEM $(0.44 \mathrm{~Hz})$

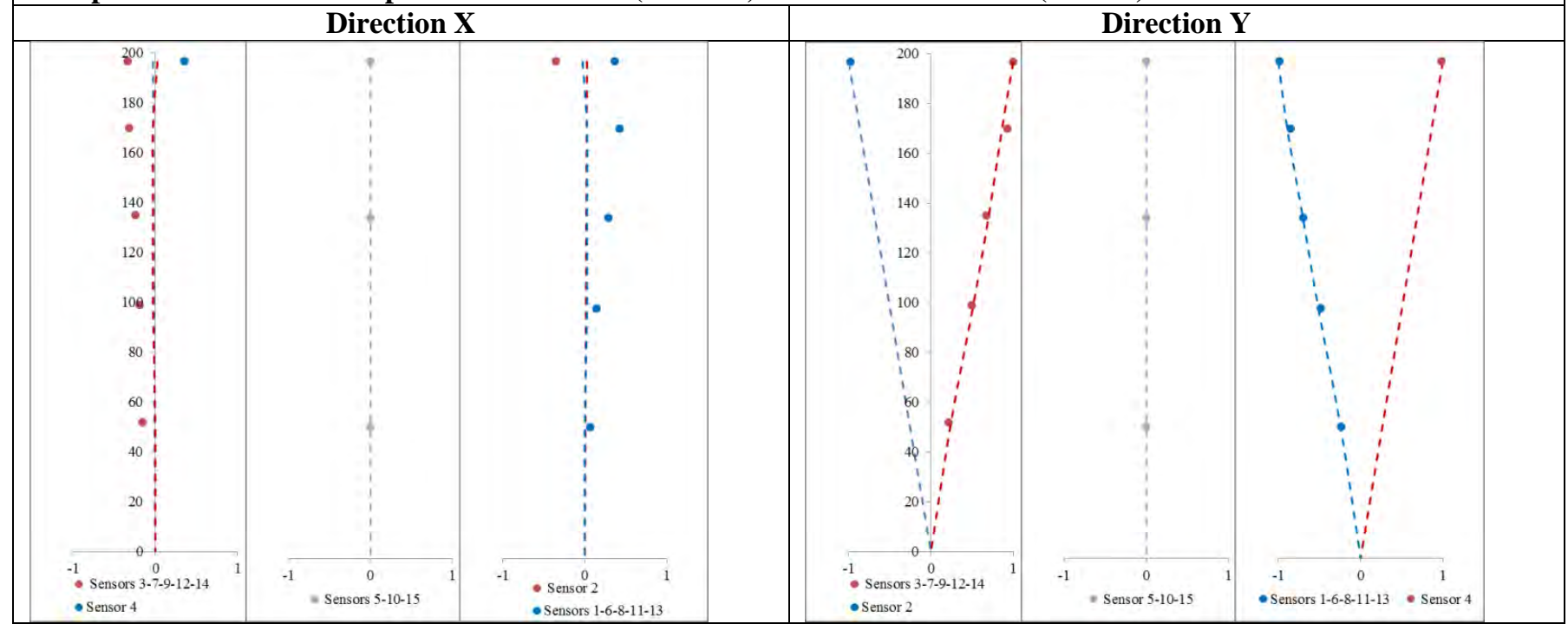

Fig. 7 - Plots of the experimental mode shapes compared to the numerical ones for Mode N.1., Mode N.2, Mode N.3 


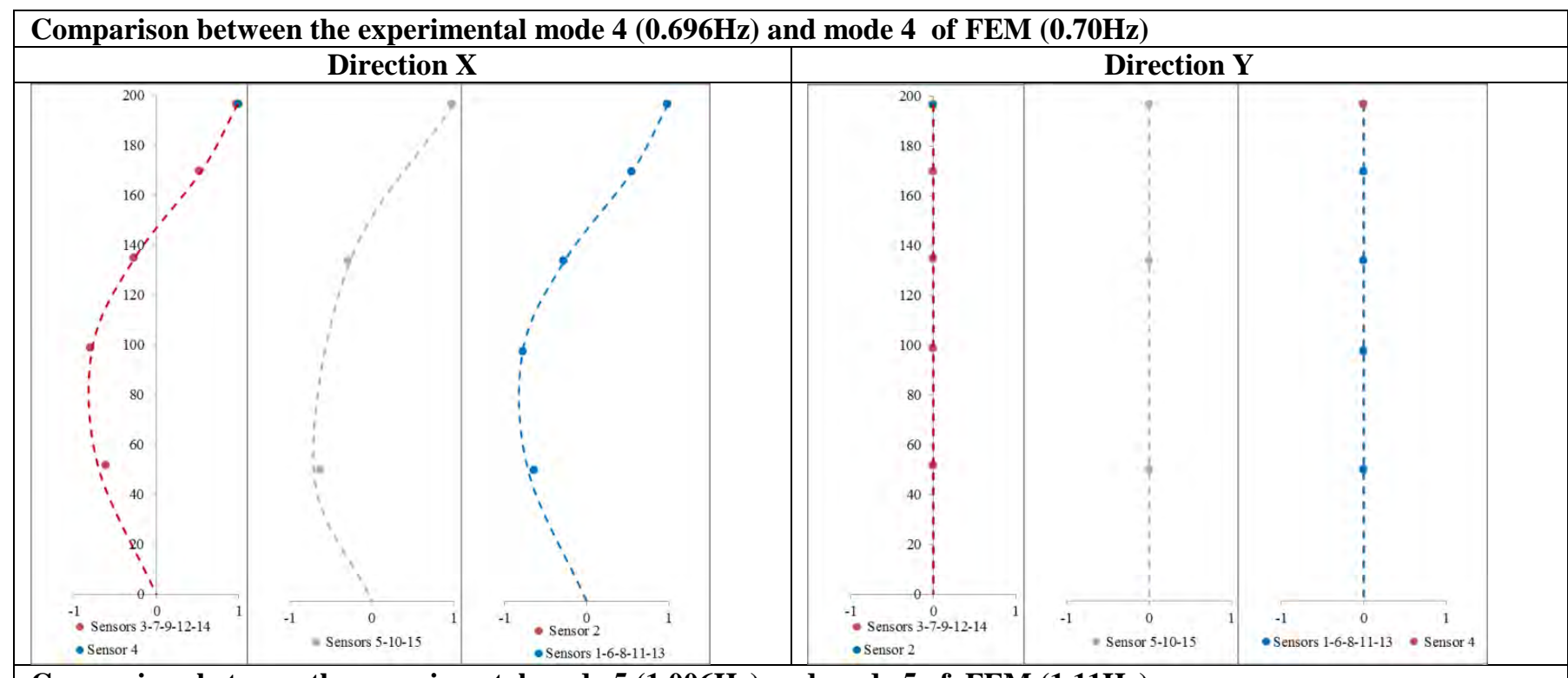

Comparison between the experimental mode $5(1.006 \mathrm{~Hz})$ and mode 5 of FEM $(1.11 \mathrm{~Hz})$

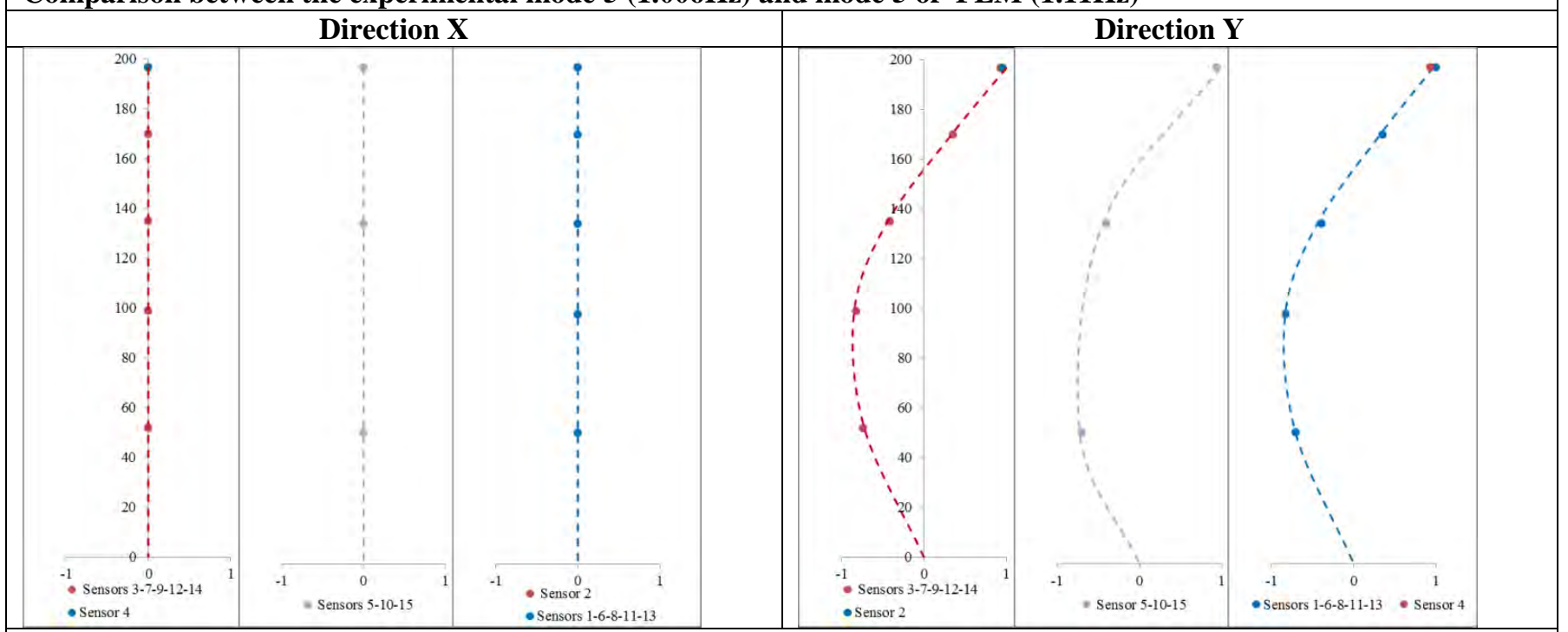

Comparison between the experimental mode $6(1.417 \mathrm{~Hz})$ and mode 7 of FEM $(1.58 \mathrm{~Hz})$ Direction X

\section{Direction Y}

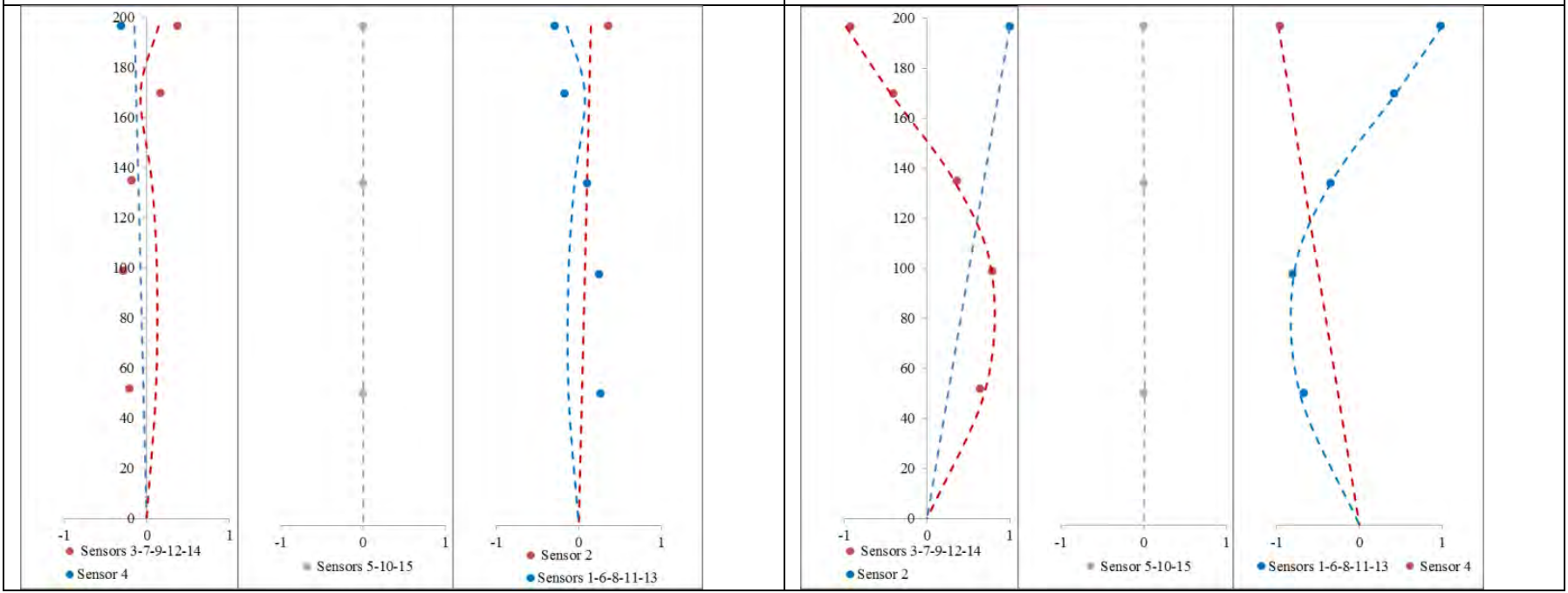

Fig.8 - Plots of the experimental mode shapes compared to the numerical ones for Mode N.4., Mode N.5, Mode N.6 


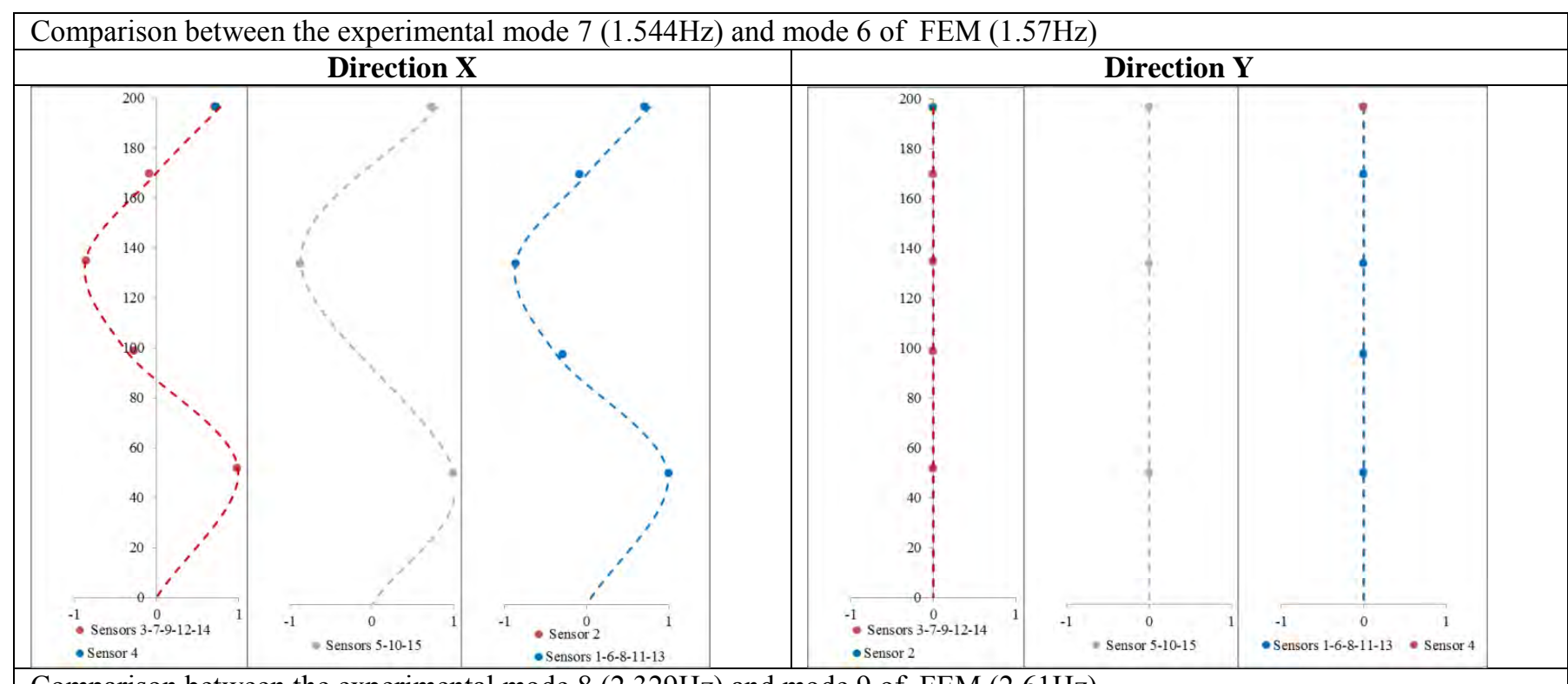

Comparison between the experimental mode $8(2.329 \mathrm{~Hz})$ and mode 9 of FEM $(2.61 \mathrm{~Hz})$

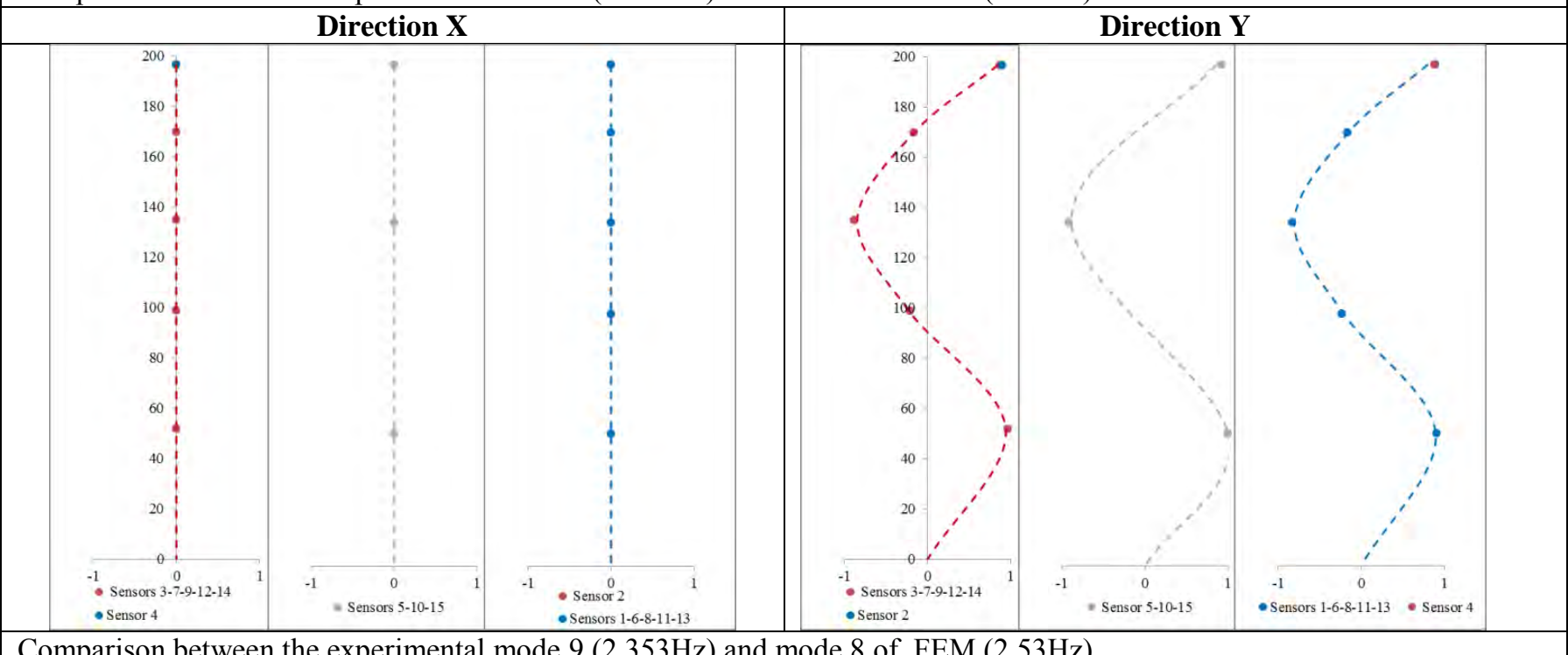

Comparison between the experimental mode $9(2.353 \mathrm{~Hz})$ and mode 8 of FEM $(2.53 \mathrm{~Hz})$

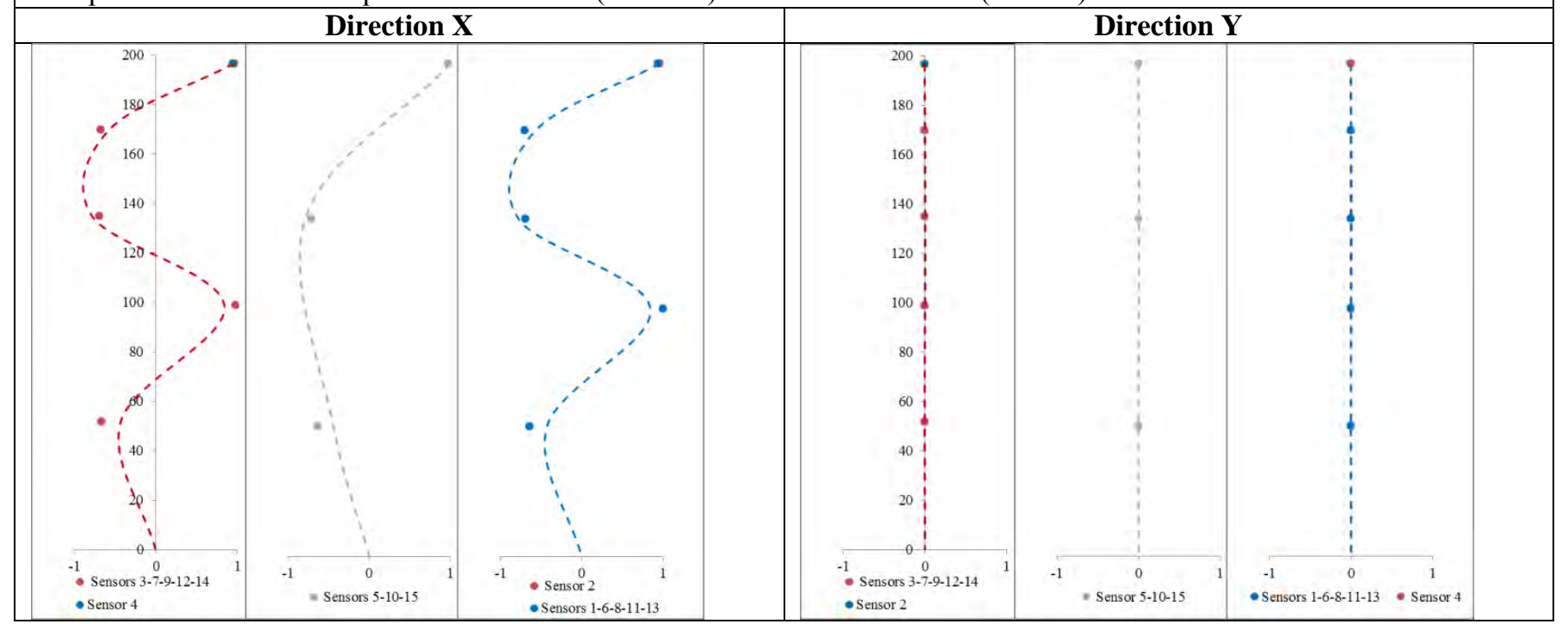

Fig.9 - Plots of the experimental mode shapes compared to the numerical ones for Mode N.7., Mode N.8, Mode N.9 


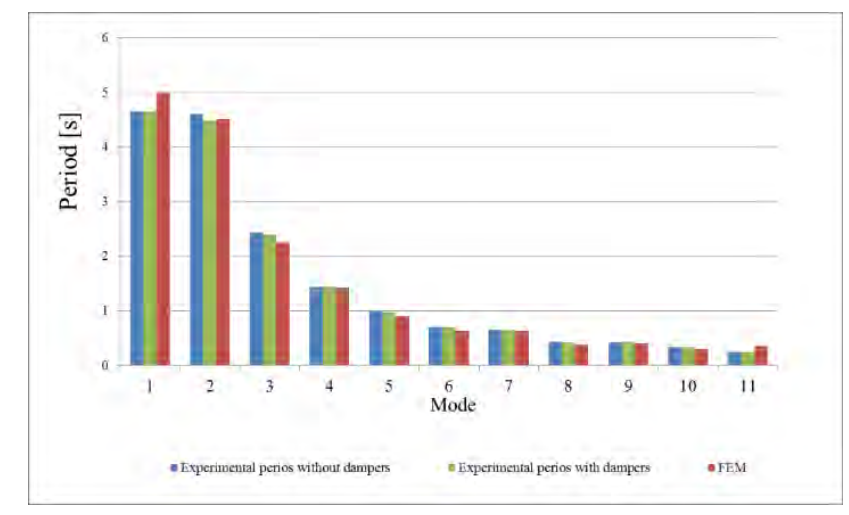

Fig.10 - Synoptic comparison of periods for the identified modes: OMA tests without dampers (blue plot), OMA test with dampers (green plot), numerical analysis (red plot)

From Tab.2 and Fig.7-9, a very good agreement between the prediction of the numerical model and the experimental results can be observed: the maximum difference between the frequencies is $12 \%$ for Mode N.8. For all the modes but Mode N.1, the numerical frequencies are slightly higher than the experimental ones, since the model was implemented using un-cracked gross sections for all the elements. The numerically derived eigenvectors are highly coherent with the values derived from the experiments, at the locations of the sensors, and for all the identified modes.

\subsection{Damping estimation with and without additional dampers}

As discussed above, the OMA tests in the two configurations were useful to validate the design and installation process of the dampers, represented in Fig. 11a, and to gauge their efficiency in service life conditions, for very low levels of vibration.

The modal damping estimations provided by the tests with the dampers are reported in Tab.3 and compared to those obtained in the configuration without dampers, which had already been reported in Tab.2. It can be observed that the dampers increase the modal damping for Mode N.2 and Mode N.3 by 3.1 times and 2.9 times respectively. These estimations proved that the design pre-requirements, i.e. for the dampers to contribute to the damping of selected modes only, i.e. Mode N.2 and Mode N.3, were achieved. Also, in Fig. 11b, the plots representing the theoretical behavior curves for the dampers are reported. On the vertical axis, the theoretical additional damping on Mode N.2 is plotted vs the values of the C constant defining the different operational conditions of the dampers: the dotted line refers to the prediction of the additional damping for the dampers in test conditions; from the latter, it can be noted that the theoretical predicted value for $\mathrm{C}=160[\mathrm{kNs} / \mathrm{mm} / \mathrm{VD}]$ is very close to the actual measured performance point in tests conditions, represented by the red dot.

By means of these remarks, an additional validation of the damping device was achieved: the devices had already undergone extensive laboratory tests according to the requirements of European Standards for fatigue and cyclic loading, but the OMA tests offered the unique opportunity to have an insight on their operational behavior, once installed on site.

Tab.3 - Experimental modal damping estimates in the configuration with and without dampers

\begin{tabular}{|l|c|c|}
\hline Exp. MODE \# & $\begin{array}{c}\text { DAMPING [\%] } \\
\text { (No dampers) }\end{array}$ & $\begin{array}{c}\text { DAMPING [\%] } \\
\text { (With dampers) }\end{array}$ \\
\hline Mode N.1 & $\mathbf{0 . 5 0}$ & $\mathbf{0 . 5 8}$ \\
\hline Mode N.2 & $\mathbf{0 . 4 8}$ & $\mathbf{1 . 5 0}$ \\
\hline Mode N.3 & $\mathbf{0 . 3 5}$ & $\mathbf{1 . 0 3}$ \\
\hline Mode N.4 & $\mathbf{0 . 3 0}$ & $\mathbf{0 . 4 0}$ \\
\hline Mode N.5 & $\mathbf{0 . 5 2}$ & $\mathbf{0 . 7 7}$ \\
\hline
\end{tabular}


a.
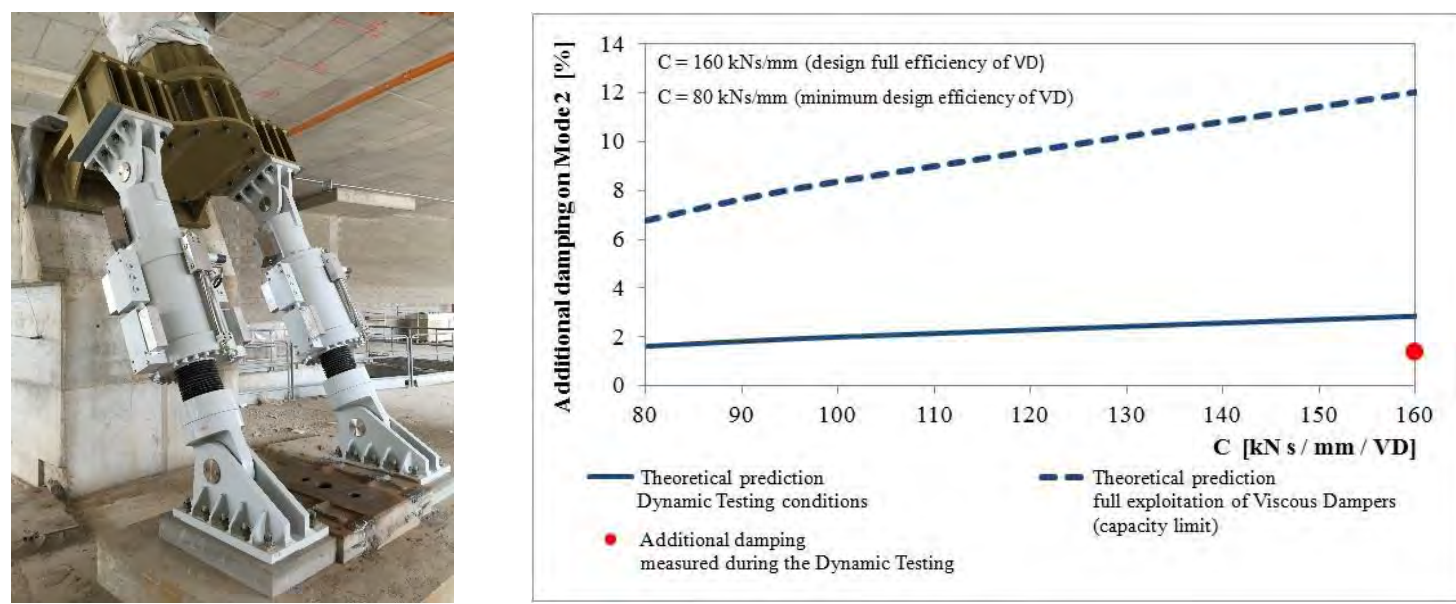

b.

Fig.11 - a. Dampers installed at the base of the struts of the Allianz Tower; b. Plots of the theoretical expected behavior of the dampers

\section{Conclusions}

The Operational Modal Analysis carried out on the recently topped-out Allianz Tower in Milan proved to be an effective tool to validate the design and construction process.

Experimental tests were carried out in two different configurations, with and without external viscous dampers, which have been designed to mitigate wind effects in service life conditions, thus allowing a better insight on the operational behavior of the devices to be achieved.

The experimentally derived modal properties are in very good agreement with the ones derived by numerical analysis, both in terms of frequency, mode shapes and sequence of modes, with the largest frequency difference being well under the $10 \%$ threshold for most of the identified modes. These results allowed a validation of the finite element model to be achieved and a reliable benchmark to be obtained for the actual continuous monitoring activity, which will allow to efficiently schedule ordinary maintenance activities and to promptly decide on the operability of the building in the aftermath of unexpected events.

\section{References}

[1] Consiglio Superiore dei Lavori Pubblici, Nuove Norme Tecniche per le Costruzioni, D.M. 14-01-2008, Roma, Italy, 2008 (in Italian)

[2] Cigada, A., Mola, E., Mola, F., Stella, G. and Vanali, M., Dynamic Behavior of the Palazzo Lombardia Tower: Comparison of Numerical Models and Experimental Results, J. of Performance of Constructed Facility, May-June, 2014

[3] Peeters, B., Van Der Auwerarer, H., Guillaume, P. and Leuridan, J., The Polymax frequency-domain method: a new standard for modal parameter estimation?, Shock and Vibration, 11(3-4), IOS Press, 2004 